Original article

\title{
The effects of transcranial direct current stimulation compared to standard bupropion for the treatment of tobacco dependence: A randomized sham-controlled trial
}

\author{
Shahram Ghorbani Behnamª , Seyed Abbas Mousavi ${ }^{\mathrm{b}}$, Mohammad Hassan Emamian,* \\ a Student Research Committee, School of Medicine, Shahroud University of Medical Sciences, Shahroud, Iran \\ b Center for Health Related Social and Behavioral Sciences Research, Shahroud University of Medical Sciences, Shahroud, Iran \\ ' Department of Epidemiology, School of Public Health, Shahroud University of Medical Sciences, Shahroud, Iran
}

\section{A R T I C L E I N F O}

\section{Article history:}

Received 10 January 2019

Received in revised form 25 April 2019

Accepted 28 April 2019

Available online 14 May 2019

Keywords:

Dorsolateral prefrontal cortex

tDCS

Smoking

Relapses

Iran

\begin{abstract}
A B S T R A C T
Background: Current treatments for smoking cessation are not effective for most smokers. This study aims to examine the effectiveness of transcranial Direct Current Stimulation (tDCS) on smoking cessation. Methods: In this randomized, sham-controlled trial study, tobacco-dependent (by DSM-5) male participants were recruited from the general public invitation. Participants were randomly allocated to 5 groups; (A), treatment with $300 \mathrm{mg}$ bupropion for 8 weeks; (B), active tDCS (20 sessions for 4 weeks); (C), sham for group B ; (D), active tDCS (20 sessions for 12 weeks), and (E), sham for group D. The electrode montage was anode F3 and cathode F4. Study outcomes include salivary cotinine, Fagerstrom test for nicotine dependence, and smoked cigarette per day, were examined on three time points. Repeatedmeasures analysis of variances and the generalized estimation equation (GEE) model were employed for data analysis.

Results: Among 210 volunteers, 170 participants completed the study. Mean age of participants was 42.9 years, ranging from 21 to 64 years. The 6-month point abstinence rates in groups A, B and D were $20 \%, 7 \%$ and $25.7 \%$, and in C, D sham groups were 3.1\% and 3\% respectively. Results of the GEE model showed that although group D was not different from group A in abstinence rate, i.e., salivary cotinine $>4(p=0.266)$, nicotine dependency by Fagerstrom test was lower in this group compared to group A $(p=0.019)$.

Conclusions: The 12-week tDCS had a clinically good therapeutic effect on smoking cessation and its dependency. It may be a substitute for bupropion treatment.
\end{abstract}

(C) 2019 Elsevier Masson SAS. All rights reserved.

\section{Introduction}

Tobacco accounts for over 7 million deaths annually [1]. Counseling and medication can increase more than double the success rate for quitting smoking [1]. About $62 \%$ of those who attempt to quit smoking on their own with no pharmacotherapy had a relapse in the first two weeks, and only $5 \%$ succeed to quit smoking after a year [2]. Smoking cessation is useful at any age for preventing the risk of co-morbidities progression and early mortality [3]. Current smoking cessation methods include counseling [4,5], and nicotine replacement therapy (chewing gum, inhalers, and nicotine patches), with bupropion and Varenicline [6]. Nevertheless, many smokers using these

\footnotetext{
* Corresponding author.

E-mail address: emamian@shmu.ac.ir (M.H. Emamian).
}

treatments also fail to quit smoking and maintain abstinence for at least six months [6-8].

Over the past decade, transcranial Direct Current Stimulation (tDCS) has been employed as a safe [9], non-invasive brain stimulation method, for addiction treatment and research on this topic is expanding [10]. Research on the effect of tDCS on smokers was mainly focused on the reduction of cue-induced craving [11,12], ability to resist smoking [13], reduction of negative affect but not cigarette craving in overnight abstinent smokers [14], and motivate smokers to quit smoking [15]. Furthermore, with repeated five sessions of stimulation per week, its cumulative effect decreased craving and even the number of cigarettes smoked [16]. In other studies, anodal stimulation with tDCS has also decreased craving for alcohol and marijuana $[17,18]$. Treatment of patients with depression with tDCS revealed maximum therapeutic effect after 20 sessions of stimulation ( 5 sessions per week) in 4 weeks [19]. On the best of our knowledge, no study has so far examined the long-term clinical effects of tDCS on smoking 
cessation, nicotine dependency, and relapse rate on a 6-month follow-up. Bupropion was approved in the US in 1997 as a smoking cessation treatment [20]. Moreover, the Department of Health and Human Services (DHHS) guidelines for the treatment of nicotine dependence recommended bupropion in 2008 as the first line of treatment for smoking cessation [21]. Therefore in this study the effectiveness of 20 sessions tDCS in two regimes ( 4 weeks \& 12 weeks) has evaluated and compared with bupropion, on the treatment of tobacco dependence with a 6-month follow-up.

\section{Methods}

The present study is a randomized sham-controlled parallelgroup clinical trial.

The participants were selected from among those responding to a public invitation for cigarette cessation. They were all righthanded, aged 15-65 years, addicted to cigarette nicotine based on DSM-5 criteria, without underlying physical or psychological disease based on interviews with a psychiatrist and WHO Disability Assessment Schedule 2.0 [22], and interested in quitting smoking. Exclusion criteria were the consumption of drugs containing sodium, potassium, and calcium; epilepsy; malnutrition; history of neurosurgery; consumption of any other addictive substance except nicotine, and the use of other nicotine-containing products such as pipe, hookah, or cigars. All of the participants refrain from smoking $2 \mathrm{~h}$ before baseline measurement and tDCS sessions during the study. This study has been carried out in accordance with The Code of Ethics of the World Medical Association (Declaration of Helsinki) for experiments involving humans. The participants were informed by a clinical psychologist of all therapeutic steps, duration of the study, randomized nature of interventions, and possible complications of interventions, and entered the study upon signing informed consent forms approved by the Ethics Committee (Ethics Code: IR.SHMU.REC.1395.75) of Shahroud University of Medical Sciences.

\subsection{Interventions}

Interventions were provided in five groups: A) pharmacotherapy, using $300 \mathrm{mg}$ bupropion (GlaxoSmithKline 2006) for 8 weeks; B) tDCS (Soterix $1 \times 1$ tDCS) for 20 sessions over 4 weeks, ( 5 sessions per week); C) The sham tDCS for 20 sessions over 4 weeks, ( 5 sessions per week); D) active tDCS for 20 sessions over 12 weeks, ( 5 sessions per week for two weeks, followed by one session per week), and E) The sham tDCS for 20 sessions over 12 weeks, ( 5 sessions per week for 2 weeks, followed by one session per week). The electrode placement montage was anode F3 and cathode F4 in tDCS groups based on standard 20/20 EEG system. Stimulation intensity was $2 \mathrm{~mA}$ for $20 \mathrm{~min}$ in tDCS groups and only $30 \mathrm{~s}$ in sham groups. This short period is the time when the device becomes ready to start the $2 \mathrm{~mA}$ stimulation. Carbon electrodes, placed inside sponges smeared with saline solution, were utilized. Anode and cathode size was 35 and $100 \mathrm{~cm}^{2}$, respectively.

\subsection{Outcomes}

Primary outcome of this study was abstinence at 6-months confirmed by salivary cotinine $<4 \mathrm{ng} / \mathrm{ml}$. Nicotine dependence severity confirmed by Fagerstrom test for nicotine dependence (FTND) and self-reported cigarette per day (CPD) were secondary and tertiary outcomes respectively. All outcomes measured at time points of baseline (time 1), post-intervention (time 2), and 6month follow-up (time 3). The length of follow-up was the same for all groups ( 6 months after the beginning of interventions). Point abstinence prevalence was calculated by the percentage of individuals with salivary cotinine of lower or equal to $4 \mathrm{ng} / \mathrm{ml}$ at different time points.

\subsection{Sample size}

Given that other studies [23-25], reported a 31\% abstinence following treatment with bupropion at six-month follow up, $\alpha=0.05, \beta=0.2$ (95\% confidence interval and $80 \%$ power), and $5 \%$ quit rate with tDCS; the sample size was calculated 35 per groups. The Sample size was smaller for salivary cotinine and Fagerstrom score which were evaluated based on mean scores of these outcomes. Therefore, the sample size was considered as 35 per group.

\subsection{Randomization}

Random allocation was designed by a nurse using a similar balls method. In this method, 175 similar balls were prepared, and the names of the five groups (A-D) were placed inside balls. Then, each participant selected one ball, thus selecting their group randomly.

\subsection{Blinding}

Interventions were conducted by one researcher who was not blinded, while salivary cotinine measurement was taken by a colleague working at the laboratory who was blinded to participant's groups. In addition, questionnaires were completed by the participants and handed to the psychologist of the group who was also blind to group classification. All data were given to the analyzer, who was blinded to the type of interventions. The participants were unaware of the type of intervention in all active and sham tDCS groups.

\subsection{Instruments}

1: The Expiration Carbon Monoxide ( $\mathrm{CO}$ ) was measured by Smokerlyzer (Bedfont Scientific Ltd, UK); each participant with CO more than 9 ppm was classified as a smoker [26]. 2: Fagerstrom Test for Nicotine Dependence (FTND) is a reliable and valid scale for determining the severity of nicotine dependence $[27,28]$, and results vary from 0 to 10 . 3: Salivary cotinine measurement with ELISA method is a very sensitive quantitative test for estimating the status of cigarette smoking in the past week [29,30]. Salivary cotinine level in non-smokers is less than or equal to $4 \mathrm{ng} / \mathrm{ml}$.

This clinical trial was registered in the Iranian Registry of Clinical Trials (registered code: IRCT2016072629093N1).

\subsection{Statistical methods}

After examining the normality of variables using ShapiroWilk's test and skewness and kurtosis indices, data were analyzed using repeated-measures analysis of variances and the generalized estimation equation (GEE) model. Maximum alpha error for hypothesis testing was determined as $0.05(\mathrm{p}<0.05)$.

\section{Results}

In this study, 210 individuals volunteered following a general invitation. 10 persons did not meet the inclusion criteria; 25 did not accept terms of research, including pharmacotherapy, tDCS, and 6month follow-up, and 5 did not visit during the intervention and follow-up due to work-related problems. Finally, 33 were allocated to Group C, 32 to Group D, and 35 to other groups, with 170 participants completing all steps of the study. Fig. 1 illustrates the number of participants in the five groups over different steps, from inclusion to analysis. Recruitment occurred from August 2016 to 


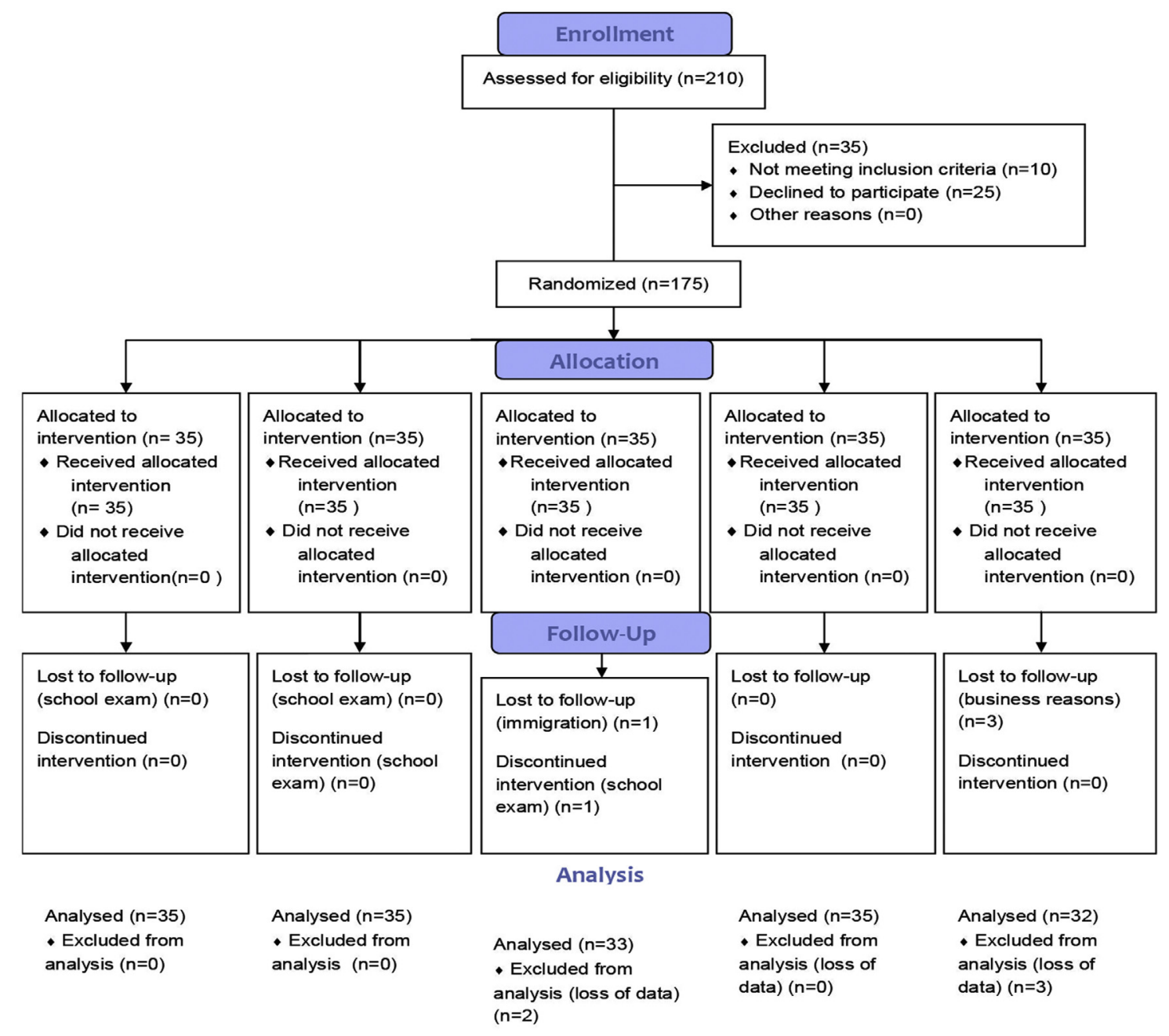

Fig. 1. Flow diagram of study.

November 2016, and interventions and follow-up lasted until August 2017.

Mean \pm SD of participants' age was $42.88 \pm 10.96$ years, ranging from 21 to 64 years. All participants were male, with 121 (71.2\%) being married, 75 (44.1\%) with high school-level education, and 114 (67.0\%) being employed. Baseline demographic characteristics of the participants in different groups are reported in Table 1.

The comparison of participants succeeding in completely quitting smoking (Salivary Cotinine $<=4$ ) at each time point (point abstinence rate) revealed that 43 people (25.3\%) succeeded in the first month and $20,(11.8 \%)$ in the six months after intervention. Examination of this status in different intervention groups at the 6month follow-up indicated that 7 participants (20\%) in Groups A, 2 participants $(5.7 \%)$ in group B, one participant $(2.8 \%)$ in Groups $C$ and $\mathrm{E}$, and 9 participants (25.7\%) in Groups D successfully quit smoking. Therefore, treatment success was markedly higher in Groups A and D compared to other groups $(p<0.001)$. Results of the GEE model in Table 2 revealed that failure in complete smoking cessation was higher in Groups $\mathrm{C}, \mathrm{E}$, and $\mathrm{B}$, compared to $\mathrm{A}$ $(p<0.001)$ and there was no statistically significant difference between groups $A$ and $D(p=0.266)$.

Results of repeated-measures ANOVA for salivary cotinine revealed that this value showed a significant difference at threetime points (baseline, post-intervention, and follow-up) ( $F=60.3$, $\mathrm{df}=2, \mathrm{P}<0.001)$. Moreover, the value of salivary cotinine differed across groups $(\mathrm{F}=6.5, \mathrm{df}=4, \mathrm{P}<0.001)$. Beside, an interaction effect exists for the values of cotinine in groups by time points $(F=9.1$, $\mathrm{df}=8, \mathrm{P}<0.001)$. As the time and group interaction effect was significant, further tests (Tables 3 and 4 ) revealed that the cotinine level differed across Groups A, B, and D in different time points $(\mathrm{P}<0.001)$. On the other hand, it was not significant in Groups $C$ $(p=0.859)$ and $E(p=0.876)$. Examination of the effect of different interventions at three- time points showed no significant difference in baseline measurements (time 1) of cotinine level across groups $(\mathrm{F}=1.8, \mathrm{df}=4, \mathrm{P}=0.130)$, which was expected considering randomization. On the second and third time points, however, cotinine level differed across groups $(\mathrm{P}<0.001)$. Table 3 presents the effect of interventions at three- time points. Examination of the trend of changes in cotinine over different periods showed that, in Groups A, B, and D, a significant linear and quadratic trend exists for cotinine levels $(p<0.001)$. Nevertheless, these trends were not significant for the sham groups (C, E). A significant increase in salivary cotinine existed in group $A$, while in groups $B$ and $D$ there were an steady state between times 2 and 3 (Table 3 and Fig. 2).

Further inter groups analysis revealed that compared to group A, cotinine levels were higher in other groups at times 2 and 3 (Table 4). Similarly, results of the GEE model, adjusted with other independent variables, including education level and age revealed that the levels of cotinine were higher in different intervention groups compared to Group A (Table 2). This model also showed that an increase in education level leads to a decrease in cotinine level $(p=0.009)$.

Results of repeated-measures ANOVA for FTND revealed that this value showed a significant difference across the three- time 
Table 1

Demographic characteristics of the participants $(n=170)$.

\begin{tabular}{|c|c|c|c|c|c|c|}
\hline \multirow[t]{2}{*}{ Independent Variables } & \multicolumn{5}{|l|}{ Groups } & \multirow[t]{2}{*}{ Total $(n=170) n(\%)$} \\
\hline & $A(n=35) n(\%)$ & $\mathrm{B}(\mathrm{n}=35) \mathrm{n}(\%)$ & $C(n=33) n(\%)$ & $\mathrm{D}(\mathrm{n}=35) \mathrm{n}(\%)$ & $E(n=32) n(\%)$ & \\
\hline \multicolumn{7}{|l|}{ Marital status } \\
\hline Single & $7(20.0)$ & $8(22.9)$ & $8(24.2)$ & $2(5.7)$ & $7(21.9)$ & $32(18.8)$ \\
\hline Married & $25(71.4)$ & $24(68.6)$ & $19(57.6)$ & $33(94.3)$ & $20(62.5)$ & $121(71.2)$ \\
\hline Divorced & $3(8.6)$ & $3(8.5)$ & $6(18.2)$ & - & $5(15.6)$ & $17(10.0)$ \\
\hline \multicolumn{7}{|l|}{ Age groups } \\
\hline $21-30$ & $3(8.6)$ & $4(11.4)$ & $5(15.2)$ & $2(5.7)$ & $5(15.6)$ & $19(11.2)$ \\
\hline $31-40$ & $11(31.4)$ & $12(34.3)$ & $7(21.2)$ & $15(42.8)$ & $13(40.6)$ & $58(34.1)$ \\
\hline $41-50$ & $9(25.7)$ & $9(25.7)$ & $11(33.3)$ & $8(22.9)$ & $6(18.8)$ & $43(25.3)$ \\
\hline$>50$ & $12(34.3)$ & $10(28.6)$ & $10(30.3)$ & $10(28.6)$ & $8(25.0)$ & $50(29.4)$ \\
\hline \multicolumn{7}{|l|}{ Education } \\
\hline Elementary & $3(8.6)$ & $3(8.6)$ & $6(18.2)$ & $6(17.1)$ & $2(6.2)$ & $20(11.8)$ \\
\hline Guidance & $5(14.3)$ & $4(11.4)$ & $5(15.1)$ & $10(28.6)$ & $6(18.8)$ & $30(17.6)$ \\
\hline High school & $18(51.4)$ & $20(57.1)$ & $13(39.4)$ & $9(25.7)$ & $15(46.9)$ & $75(44.1)$ \\
\hline University & $9(25.7)$ & $8(22.9)$ & $9(27.3)$ & $10(28.6)$ & $9(28.1)$ & $45(26.5)$ \\
\hline \multicolumn{7}{|l|}{ Job } \\
\hline Unemployed & $5(14.3)$ & $5(14.3)$ & $3(9.1)$ & $6(17.1)$ & $8(25.0)$ & $27(15.8)$ \\
\hline Employed & $26(74.2)$ & $25(71.3)$ & $21(63.6)$ & $26(74.3)$ & $16(50.0)$ & $114(67.0)$ \\
\hline Student & $1(2.9)$ & $1(2.9)$ & $1(3.0)$ & - & - & $3(1.8)$ \\
\hline Retired & $3(8.6)$ & $3(8.6)$ & $5(15.2)$ & $3(8.6)$ & $5(15.6)$ & $19(11.2)$ \\
\hline Other & - & $1(2.9)$ & $3(9.1)$ & - & $3(9.4)$ & $7(4.2)$ \\
\hline
\end{tabular}

Table 2

The effects of different interventions on various outcomes in Generalized Estimating Equation models.

\begin{tabular}{|c|c|c|c|c|c|c|c|c|}
\hline \multirow[t]{2}{*}{ Groups } & \multicolumn{2}{|l|}{ Salivary Cotinine } & \multicolumn{2}{|l|}{ FTND } & \multicolumn{2}{|l|}{$\mathrm{CPD}$} & \multicolumn{2}{|l|}{ Salivary Cotinine $>4$} \\
\hline & Coefficient (95\% CI) & $P$ value & Coefficient (95\% CI) & $P$ value & Coefficient (95\% CI) & $P$ value & Coefficient (95\% CI) & $P$ value \\
\hline A & Reference & - & Reference & - & Reference & - & Reference & - \\
\hline B & $48.9(18.4-79.4)$ & 0.002 & $-0.1(-0.9$ to 0.6$)$ & 0.754 & $6.7(2.4-11.0)$ & 0.002 & $0.5(-0.6$ to 1.5$)$ & 0.373 \\
\hline C & $61.2(30.3-92.1)$ & $<0.001$ & $1.1(0.3-1.8)$ & 0.006 & $8.7(4.2-13.1)$ & $<0.001$ & $2.7(0.4-5.0)$ & 0.022 \\
\hline $\mathrm{D}$ & $41.4(10.5-72.3)$ & 0.009 & $-1.0(-1.7$ to -0.1$)$ & 0.019 & $8.8(4.5-13.3)$ & $<0.001$ & $-0.5(-1.4$ to 0.4$)$ & 0.266 \\
\hline E & $79.1(47.7-110.6)$ & $<0.001$ & $-0.4(-1.2$ to 0.3$)$ & 0.283 & $12.7(8.2-17.3)$ & $<0.001$ & $2.6(0.3-4.9)$ & 0.029 \\
\hline Education (Year) & $-4.2(-7.4$ to -1.1$)$ & 0.009 & $-0.1(-0.2$ to 0.02$)$ & 0.009 & $-0.5(-1.0$ to 0.0$)$ & 0.022 & $-0.1(-0.2$ to -0.01$)$ & 0.040 \\
\hline Age (Year) & $-0.3(-1.3$ to 0.8$)$ & 0.621 & $-0.01(-0.03$ to 0.01$)$ & 0.254 & $0.0(-0.2$ to 0.2$)$ & 0.879 & $-0.03(-0.1$ to 0.01$)$ & 0.100 \\
\hline
\end{tabular}

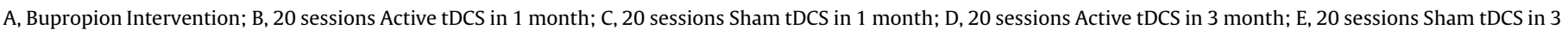
month; FTND, Fagerstrom Test of Nicotine Dependence; CPD, Cigarette Per Day; CI, Confidence Intervals.

periods $(\mathrm{F}=95.7, \mathrm{df}=2, \mathrm{P}<0.001)$. Furthermore, the value of FNDT differed across groups $(\mathrm{F}=5.9, \mathrm{df}=4, \mathrm{P}<0.001)$. Also, there was an interaction effect for this value for groups by time $(\mathrm{F}=18.9, \mathrm{df}=8$, $\mathrm{P}<0.001$ ). As the time and group interaction effect, was significant, further tests revealed that the FTND score differed across Groups B, $\mathrm{A}$, and $\mathrm{D}$ in different time points $(\mathrm{P}<0.001)$. On the other hand, it was not significant in Groups $C(p=0.850)$ and $E(p=0.995)$ (Fig. 3 ). Examination of the effect of different treatments at three- time points showed that, at all-time points, FTND scores differed across groups $(\mathrm{p}<0.001)$.

Examination of the trend of change in FTND over different time periods indicated that, in Groups A, B, and D, a significant linear and quadratic trend existed $(p<0.001)$. None of these trends was significant in sham groups. Further analysis revealed that compared to group A, FTND were different in other groups at different time points, except for group B (Table 4). Similarly, results of the GEE model, adjusted with other independent variables (Table 2), showed that dependence severity did not differ in Groups B, and E compared to Group A but differed in Groups C and D compared to Group A. This model also showed that an increase in education level lead to reduction in nicotine dependence $(p=0.009)$, but age had no such effect $(p=0.254)$.

Results of repeated-measures ANOVA for CPD showed a significant difference across the three- time periods $(F=81.3$, $\mathrm{df}=2, \mathrm{P}<0.001)$. Moreover, the value of $\mathrm{CPD}$ differed across groups $(\mathrm{F}=8.1, \mathrm{df}=4, \mathrm{P}<0.001)$. Also, there was an interaction effect for this value for groups by time $(\mathrm{F}=11.3, \mathrm{df}=8, \mathrm{P}<0.001)$. As the time and group interaction effect, was significant, further tests revealed that CPD differed across Groups A, B, and D in different time points $(\mathrm{P}<0.001)$. On the other hand, it was not significant for Groups $C$ $(\mathrm{p}=0.770)$ and $\mathrm{E}(\mathrm{p}=0.654)$ (Fig. 4). Examination of the effect of different interventions at three- time points showed that, at alltime points, CPD differed across groups $(\mathrm{p}<0.001)$. Examination of the trend of changes in CPD over different periods showed that, in Groups A, B, and D, a significant linear and quadratic trend existed for CPD $(p<0.001)$. Further analysis revealed that compared to group A, CPD was higher in other groups at different time points (Table 4). Similarly, results of the GEE model, adjusted with other independent variables, revealed that the level of CPD differed in different intervention groups compared to Group A (Table 2). This model also indicated that an increase in education level significantly decreased the CPD $(p=0.025)$.

In this study, there were no complications reported from bupropion (except for 2 cases of insomnia and 3 cases of dry mouth) or tDCS ( 4 cases of the mild headache) to motivate participants to withdraw from interventions. All the noted minor side effects were resolved without pharmacotherapy.

\section{Discussion}

This study investigates two different tDCS protocols with their sham controls and compares these to standard treatment for tobacco dependence. The main finding of the study was that the longer duration tDCS protocol (20 sessions over 12 weeks: 2 weeks 
Table 3

The effects of interventions on outcomes in different times in repeated measure analysis of variance models.

\begin{tabular}{|c|c|c|c|c|c|c|c|}
\hline \multirow[t]{2}{*}{ Groups } & \multirow[t]{2}{*}{ Times } & \multicolumn{2}{|l|}{ Salivary Cotinine $(\mu \mathrm{ml} / \mathrm{dl})$} & \multicolumn{2}{|l|}{ FTND } & \multicolumn{2}{|l|}{ CPD } \\
\hline & & Coefficient (95\% CI) & $P$ value & Coefficient (95\% CI) & P value & Coefficient (95\% CI) & $P$ value \\
\hline \multirow[t]{3}{*}{ A } & 2 vs 1 & $-95.5(-116.2$ to -74.8$)$ & $<0.001$ & $-4.4(-5.1$ to -3.7$)$ & $<0.001$ & $-10.7(-13.1$ to -8.3$)$ & $<0.001$ \\
\hline & 3 vs 1 & $-71.8(-92.5$ to -51.1$)$ & $<0.001$ & $-3.2(-3.9$ to -2.5$)$ & $<0.001$ & $-11.3(-13.7$ to -8.9$)$ & $<0.001$ \\
\hline & 3 vs 2 & $23.7(3.1-44.4)$ & 0.024 & $1.3(0.6-2.0)$ & $<0.001$ & $-0.6(-3.0$ to 1.8$)$ & 0.640 \\
\hline \multirow[t]{3}{*}{ B } & 2 vs 1 & $-57.6(-78.2$ to -36.9$)$ & $<0.001$ & $-2.7(-3.4$ to -2.0$)$ & $<0.001$ & $-9.0(-11.4$ to -6.6$)$ & $<0.001$ \\
\hline & 3 vs 1 & $-46.0(-66.7$ to -25.3$)$ & $<0.001$ & $-2.5(-3.2$ to -1.8$)$ & $<0.001$ & $-5.9(-8.3$ to -3.5$)$ & $<0.001$ \\
\hline & 3 vs 2 & $11.6(-9.1$ to 32.2$)$ & 0.274 & $0.2(-0.5$ to 0.9$)$ & 0.522 & $3.1(0.7-5.5)$ & 0.010 \\
\hline \multirow[t]{3}{*}{ C } & 2 vs 1 & $-3.9(-25.2$ to 17.4$)$ & 0.720 & $0.0(-0.7$ to 0.7$)$ & 1.000 & $-0.9(-3.4$ to 1.6$)$ & 0.470 \\
\hline & 3 vs 1 & $-5.9(-27.2$ to 15.4$)$ & 0.588 & $0.2(-0.5$ to 0.9$)$ & 0.621 & $-0.5(-3.0$ to 2.0$)$ & 0.683 \\
\hline & 3 vs 2 & $-2.0(-23.3$ to 19.3$)$ & 0.854 & $0.2(-0.5$ to 0.9$)$ & 0.621 & $0.4(-2.0$ to 2.9$)$ & 0.754 \\
\hline \multirow[t]{3}{*}{$\mathrm{D}$} & 2 vs 1 & $-86.8(-107.4$ to -66.1$)$ & $<0.001$ & $-3.2(-3.9$ to -2.5$)$ & $<0.001$ & $-10.6(-13.0$ to -8.2$)$ & $<0.001$ \\
\hline & 3 vs 1 & $-71.9(-92.6$ to -51.2$)$ & $<0.001$ & $-3.5(-4.2$ to -2.8$)$ & $<0.001$ & $-10.1(-12.5$ to -7.7$)$ & $<0.001$ \\
\hline & 3 vs 2 & $14.9(-5.8$ to 35.5$)$ & 0.159 & $-0.3(-1.0$ to 0.4$)$ & 0.337 & $0.5(-2.0$ to 2.9$)$ & 0.709 \\
\hline \multirow[t]{3}{*}{$\mathrm{E}$} & 2 vs 1 & $-3.9(-25.5$ to 17.7$)$ & 0.725 & $0.0(-0.7$ to 0.8$)$ & 0.933 & $-1.2(-3.7$ to 1.4$)$ & 0.366 \\
\hline & 3 vs 1 & $-5.5(-27.2$ to 16.1$)$ & 0.616 & $0.0(-0.7$ to 0.8$)$ & 0.933 & $-0.8(-3.3$ to 1.7$)$ & 0.541 \\
\hline & 3 vs 2 & $-1.6(-23.3$ to 20.0$)$ & 0.882 & $0.0(-0.7$ to 0.7$)$ & 1.000 & $0.4(-2.1$ to 2.9$)$ & 0.769 \\
\hline
\end{tabular}

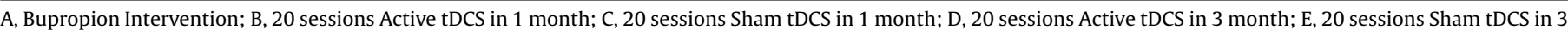
month; 1, baseline; 2, after intervention; 3, fallow up; FTND, Fagerstrom Test of Nicotine Dependence; CPD, Cigarette Per Day; CI, Confidence Intervals.

Table 4

The comparison of different interventions with group A in different times in repeated measure analysis of variance models.

\begin{tabular}{|c|c|c|c|c|c|c|c|}
\hline \multirow[t]{2}{*}{ Groups } & \multirow[t]{2}{*}{ Times } & \multicolumn{2}{|c|}{ Salivary Cotinine $(\mu \mathrm{m} / \mathrm{dl})$} & \multicolumn{2}{|l|}{ FTND } & \multicolumn{2}{|l|}{ CPD } \\
\hline & & Coefficient (95\% CI) & $P$ value & Coefficient (95\% CI) & P value & Coefficient (95\% CI) & $P$ value \\
\hline \multirow[t]{3}{*}{ B vs $\mathrm{A}$} & 1 & $28.5(-7.4$ to 64.4$)$ & 0.120 & $-0.9(-1.8$ to 0.1$)$ & 0.071 & $4.3(-0.5$ to 9.1$)$ & 0.079 \\
\hline & 2 & $66.4(30.5-102.3)$ & $<0.001$ & $0.8(-0.1$ to 1.8$)$ & 0.091 & $6.0(1.2-10.8)$ & 0.015 \\
\hline & 3 & $54.2(18.3-90.1)$ & 0.003 & $-0.2(-1.2$ to 0.8$)$ & 0.683 & $9.7(4.9-14.5)$ & $<0.001$ \\
\hline \multirow[t]{3}{*}{ C vs $A$} & 1 & $9.7(-26.7$ to 46.2$)$ & 0.600 & $-1.5(-2.5$ to -0.5$)$ & 0.003 & $1.3(-3.5$ to 6.2$)$ & 0.586 \\
\hline & 2 & $101.3(64.9-137.8)$ & $<0.001$ & $2.9(1.9-3.9)$ & $<0.001$ & $11.2(6.3-16.0)$ & $<0.001$ \\
\hline & 3 & $75.6(39.2-112.0)$ & $<0.001$ & $1.8(0.9-2.8)$ & $<0.001$ & $12.1(7.3-17.0)$ & $<0.001$ \\
\hline \multirow[t]{3}{*}{ D vs $A$} & 1 & $44.5(8.6-80.3)$ & 0.015 & $-1.0(-2.0$ to -0.1$)$ & 0.036 & $9.1(4.3-13.9)$ & $<0.001$ \\
\hline & 2 & $53.2(17.4-89.1)$ & 0.004 & $0.2(-0.8$ to 1.2$)$ & 0.683 & $9.3(4.5-14.0)$ & $<0.001$ \\
\hline & 3 & $44.4(8.5-80.2)$ & 0.016 & $-1.4(-2.4$ to -0.4$)$ & 0.004 & $10.3(5.5-15.1)$ & $<0.001$ \\
\hline \multirow[t]{3}{*}{ E vs $A$} & 1 & $26.5(-10.2$ to 63.2$)$ & 0.157 & $-2.9(-3.9$ to -2.0$)$ & $<0.001$ & $5.3(0.4-10.2)$ & 0.033 \\
\hline & 2 & $118.1(81.4-154.9)$ & $<0.001$ & $1.5(0.5-2.5)$ & 0.002 & $14.9(10.0-19.8)$ & $<0.001$ \\
\hline & 3 & $92.8(56.0-129.5)$ & $<0.001$ & $0.3(-0.7$ to 1.2$)$ & 0.598 & $15.9(10.9-20.7)$ & $<0.001$ \\
\hline
\end{tabular}

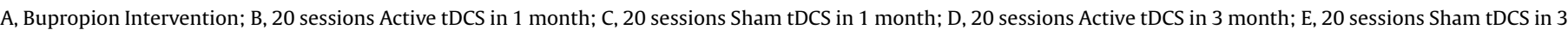
month; 1, baseline; 2, after intervention; 3, fallow up; FTND, Fagerstrom Test of Nicotine Dependence; CPD, Cigarette Per Day; CI, Confidence Intervals.

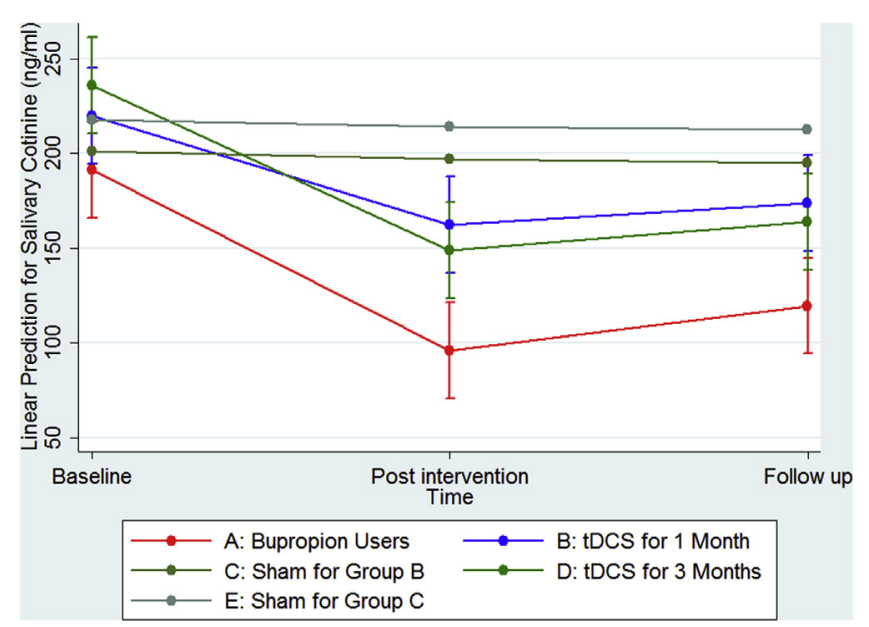

Fig. 2. Salivary Cotinine changes according to different times and groups.

of daily stimulation followed by weekly booster sessions for 10 weeks) resulted in the highest abstinence rate at 6 months (25.7\%) and was significantly more effective than the shorter stimulation protocol (20 sessions over 4 weeks) (7\%) and both sham controls. So it can be claimed that the effects of tDCS on smoking cessation are more stable in long-term interventions. The 6-month abstinence rate for standard treatment with bupropion was 20\% which did not show any significant difference with Group D (25.7\%) $(p=0.266)$. However, this difference is clinically significant and the lack of statistical significance may be attributed to the small sample size in groups.

Salivary cotinin and FTND values increased significantly during 6-month follow-up compared to post intervention measurements in bupropion users (group A), while these changes were not significant for both types of tDCS protocol (Table 3 and Fig. 2). These findings suggest that the bupropion group was relapsing to smoking whereas the tDCS groups seemed to maintain abstinence. This is important because relapse following pharmacotherapy for smoking cessation is very common.

To the best of our knowledge, this is the first study that investigates tDCS in tobacco dependence for 6-month abstinence. Therefore the comparison of results with other studies is a bit difficult. In a study in which 615 smokers treated with $300 \mathrm{mg}$ bupropion and counseling, the 6-month abstinence rate was $26.9 \%$ which was higher than placebo group [31]. Similarly, in a study on 600 adult smokers, treated with slow-release $300 \mathrm{mg}$ bupropion and counseling, the quit rate at 26 weeks was $21.0 \%$ vs. $13.7 \%$ in placebo group [32]. However, What should be considered in comparison of these studies is that counseling was not provided in the current study, and the smoking abstinence measurement scale 


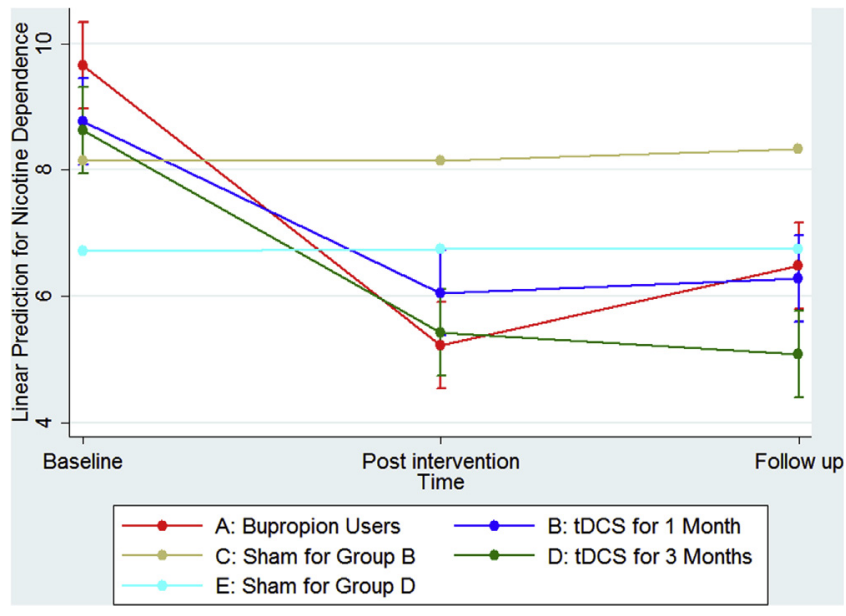

Fig. 3. Nicotine dependent severity according to different times and groups.

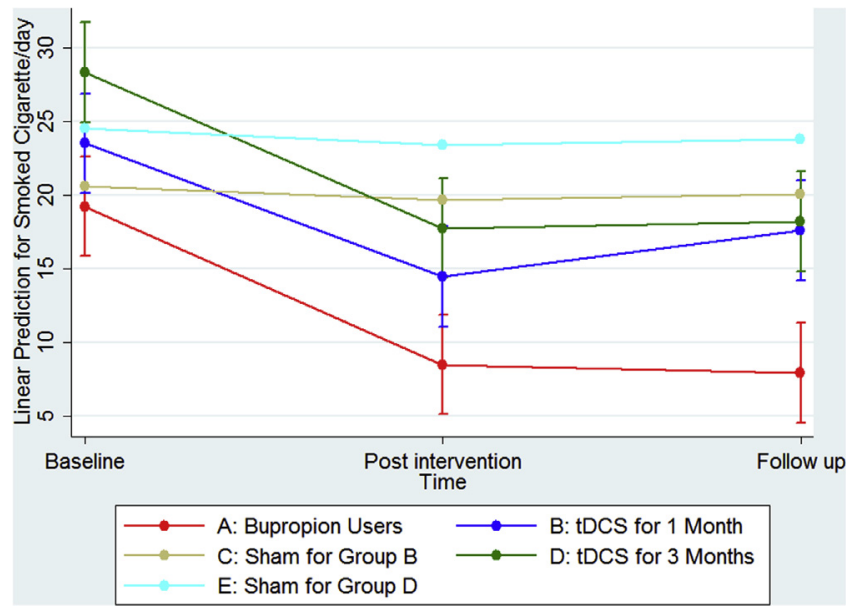

Fig. 4. Cigarette per day smoking changes according to different times and groups.

was a quantitative and more reliable method compared to most previous studies which using self-reported measurements [33]. Previous studies have shown that five F3 anodal stimulations reduce CPD by about 30\% compared to the sham group [16]. However, we did not find any study on the persistence effects of tDCS on dependence severity and CPD for six months.

Smoking cessation is related to a reduced dopaminergic activity which induces craving and relapse [34]. Although little is known about the mechanism of effect of tDCS, various theories have been proposed in this regard. Studies indicate the mediation of neuromodulators of dopamine (DA-D2) [35], and N-Methyl-DAspartic Acid (NMDA) [36], in post-effect changes (cortical excitability changes) and, consequently, long- lasting synaptic plasticity changes [37]. The severity of this plasticity effects is based on size and forms of evoked potentials stimulations [38]. Anodal stimulation increases and cathodal stimulation reduces the excitability of cortical neurons $[39,40]$. Therefore, the stimulation of dorsolateral prefrontal cortex (DLPFC) can induce the release of dopamine in mesolimbic pathways, which leads to a transient increase in dopaminergic activity and, thus, mimics the effects of substances in the mesolimbic pathways, thereby assisting the temporary reduction in craving [41]. However, it is believed that the long-term effects of tDCS on cortical excitability are due to the glutamatergic mechanism [42]. This change in neural excitability occurs at the time of stimulation and continues in the delayed form afterward, depending on the duration and intensity of stimulations as well as the person's baseline neural excitability [43]. It seems that the most crucial factor in relapse and failure of smoking cessation is negative affects in nicotine abstinence state [44,45]. But it must be noted that the anti-depressant effects of bupropion comprise less than $20 \%$ of its effects as a smoking cessation drug [46]. Human and animal studies indicate several possible cognitive mechanisms which may play a role in reducing smoking and smoking cessation by the stimulation of the DLPFC region. These include reducing nicotine craving $[11,16,41,47]$, emotion regulation and stress control $[48,49]$, decreasing high-risk behaviours [50], and enhancement of decision-making skill [51], due to the direct anodal stimulation of DLPFC regions and, therefore, subcortical synchronization activity [52].

Repeated electrical stimulation in the reduction of nicotine dependence severity (FTND) was more stable than drug treatment (Tables 3 and 4). It appears that the effects of neural adaptation and synaptic plasticity in the meso-cortico-limbic reward pathway through multiple sessions of stimulation cause this effect [53]. The lower FTND in group D compared to group A (Coefficient $=-1.0$, $\mathrm{P}=0.019$ ) (Table 2 ), may be due to the tDCS effects on the ability to resist smoking [13], emotion regulation [14] and decrease highrisk behaviours [50].

Analysis of CPD data (Table 3 ) showed a decreased value in postintervention compared to the baseline (time 2 vs. 1) and follow-up compared to the baseline (time 3 vs. 1) in Groups A, B, and D $(p<0.001)$. Nevertheless, comparison of CPD in post-intervention compared to follow-up (time 3 vs. 2) showed no significant changes for Groups A ( $\mathrm{p}=0.640)$ and $\mathrm{D}(\mathrm{p}=0.709)$ and an increased value for Group B $(p<0.010)$ (Table 3 and Fig. 4), which indicated on cumulative effects of tDCS on CPD. In previous studies, the median decrease in cigarettes at the end of 5 sessions of stimulation was $30 \%$ compared to $10 \%$ in the sham group and similar to our findings, magnitude of tDCS effects increased after each session of stimulation [13].

Finally, it is important to note that there are many intercorrelation between outcomes of this study (i.e. Cotinin, CPD and FTND) so it is not surprising that they all show the same pattern of results.

We are aware that our research has some limitations: 1 . In the past decade, the NRT treatment with Varenicline in addition to counseling has mostly been used for smoking cessation [6]. However, we used bupropion in this study as a drug intervention. 2. No woman responded to the public invitation and all participants were men. This limits the generalizability of results to the female population. 3. The sample size for some comparisons was low. Indeed, further studies on different races and with larger sample sizes are required to generalize results to all communities.

\section{Funding}

This work was supported by Shahroud University of Medical Sciences, Iran as a PhD dissertation (Project No. 9521).

\section{Decleration of interest}

The authors of this manuscript did not have any conflict of interest.

\section{Acknowledgments}

This study is a part of Ph.D. dissertation on addiction studies in Shahroud University of Medical Sciences (Project number: 9521). The authors are grateful to the deputy for research in this university and the respected participants who collaborated in this study. 


\section{References}

[1] World Health Organization. Key facts on tobacco[WHO web site]. March 9. 2018 Available at: http://www.who.int/news-room/fact-sheets/detail/tobacco [Accessed 24 June 2018].

[2] Garvey AJ, Bliss RE, Hitchcock JL, Heinold JW, Rosner B. Predictors of smoking relapse among self-quitters: a report from the Normative Aging Study. Addict Behav 1992;17(4):367-77.

[3] National Center for Chronic Disease Prevention and Health Promotion (US) Office on Smoking and Health. The health consequences of smoking-50 years of progress: a report of the surgeon general. Atlanta (GA): Centers for Disease Control and Prevention (US); 2014. . [Accessed 20 June 2018] https://www. ncbi.nlm.nih.gov/books/NBK179276/.

[4] Goldstein MG, Niaura R, Willey C, Kazura A, Rakowski W, DePue J, et al. An academic detailing intervention to disseminate physician-delivered smoking cessation counseling: smoking cessation outcomes of the Physicians Counseling Smokers Project. Prev Med 2003;36(2):185-96, doi:http://dx.doi. org/10.1016/S0091-7435(02)00018-X.

[5] Zhu SH, Stretch V, Balabanis M, Rosbrook B, Sadler G, Pierce JP. Telephone counseling for smoking cessation: effects of single-session and multiplesession interventions. J Consult Clin Psychol 1996;64(1):202-11.

[6] Heydari G, Masjedi M, Ahmady AE, Leischow SJ, Lando HA, Shadmehr MB, et al. A comparative study on tobacco cessation methods: a quantitative systematic review. Int J Prev Med 2014;5(6):673-8.

[7] Tønnesen P, Tonstad S, Hjalmarson A, Lebargy F, Van Spiegel PI, Hider A, et al. A multicentre, randomized, double-blind, placebo-controlled, 1-year study of bupropion SR for smoking cessation. J Intern Med 2003;254(2):184-92, doi: http://dx.doi.org/10.1046/j.1365-2796.2003.01185.x.

[8] Silagy C, Mant D, Fowler G, Lancaster T. Meta-analysis on efficacy of nicotine replacement therapies in smoking cessation. Lancet 1994;343(8890):139-42, doi:http://dx.doi.org/10.7326/ACPIC-1994-121-1-019.

[9] Bikson M, Grossman P, Thomas C, Zannou AL, Jiang J, Adnan T, et al. Safety of transcranial direct current stimulation: evidence based update 2016. Brain Stimul 2016;9:64161, doi:http://dx.doi.org/10.1016/j.brs.2016.06.004.

[10] Dunlop K, Hanlon CA, Downar J. Non-invasive brainstimulation treatments for addiction and major depression. Ann N Y Acad Sci 2017;1384:31-54, doi: http://dx.doi.org/10.1111/nyas.12985.

[11] Fregni F, Liguori P, Fecteau S, Nitsche MA, Pascual-Leone A, Boggio PS. Cortical stimulation of the prefrontal cortex with transcranial direct current stimulation reduces cue-provoked smoking craving: a randomized, shamcontrolled study. J Clin Psychiatry 2008;69(1):32-40, doi:http://dx.doi.org/ 10.1016/j.neubiorev.2009.11.006.

[12] Mondino M, Luck D, Grot S, Januel D, Suaud-Chagny MF, Poulet E, et al. Effects of repeated transcranial direct current stimulation on smoking, craving and brain reactivity to smoking cues. Sci Rep 2018;8(Jun (1)):8724, doi:http://dx. doi.org/10.1038/s41598-018-27057-1.

[13] Falcone M, Bernardo L, Ashare RL, Hamilton R, Faseyitan O, McKee SA, et al. Transcranial direct current brain stimulation increases ability to resist smoking. Brain Stimul 2016;9:191-6.

[14] Xu J, Fregni F, Brody AL, Rahman AS. Transcranial direct current stimulation reduces negative affect but not cigarette craving in overnight abstinent smokers. Front Psychiatry 2013;4:112.

[15] Vitor de Souza Brangioni MC, Pereira DA, Thibaut A, Fregni F, Brasil-Neto JP, Boechat-Barros R. Effects of prefrontal transcranial direct current stimulation and motivation to quit in tobacco smokers: a randomized, sham controlled, double-blind trial. Front Pharmacol 2018;9:14, doi:http://dx.doi.org/10.3389/ fphar.2018.00014

[16] Boggio PS, Liguori P, Sultani N, Rezende L, Fecteau S, Fregni F. Cumulative priming effects of cortical stimulation on smoking cue-induced craving. Neurosci Lett 2009;463(1):82-6, doi:http://dx.doi.org/10.1016/j. neulet.2009.07.041.

[17] Boggio PS, Sultani N, Fecteau S, Merabet L, Mecca T, Pascual-Leone A, et al. Prefrontal cortex modulation using transcranial DC stimulation reduces alcohol craving: a double-blind, sham-controlled study. Drug Alcohol Depend 2008;92(1-3):55-60, doi:http://dx.doi.org/10.1016/j. drugalcdep.2007.06.011.

[18] Boggio PS, Zaghi S, Villani AB, Fecteau S, Pascual-Leone A, Fregni F. Modulation of risk-taking in marijuana users by transcranial direct current stimulation (tDCS) of the dorsolateral prefrontal cortex (DLPFC). Drug Alcohol Depend 2010;112(3):220-5, doi:http://dx.doi.org/10.1016/j. drugalcdep.2010.06.019.

[19] Martin DM, Alonzo A, Mitchell PB, Sachdev P, Gálvez V, Loo CK. Frontoextracephalic transcranial direct current stimulation as a treatment for major depression: an open-label pilot study. J Affect Disord 2011;134(1):459-63, doi: http://dx.doi.org/10.1016/j.jad.2011.05.018.

[20] Foulds J, Burke M, Steinberg M, Williams JM, Ziedonis DM. Advances in pharmacotherapy for tobacco dependence. Expert Opin Emerg Drugs 2004;9 (May (1)):39-53, doi:http://dx.doi.org/10.1517/eoed.9.1.39.32951.

[21] Fiore MC, Jaén CR, Baker TB, Bailey WC, Benowitz NL, Curry SJ, et al. Treating tobacco use and dependence: update. Clinical Practice Guideline. Rockville, MD: U.S. Department of Health and Human Services. Public [210_TD\$DIFF] Health Service; 2008.

[22] Gold LH. DSM-5 and the assessment of functioning: the World Health Organization Disability Assessment schedule 2.0 (WHODAS 2.0). J Am Acad Psychiatry Law 2014;42:173-81.
[23] Aubin HJ, Lebargy F, Berlin I, Bidaut-Mazel C, Chemali-Hudry J, Lagrue G. Efficacy of bupropion and predictors of successful outcome in a sample of French smokers: a randomized placebo-controlled trial. Addiction 2004;99 (9):1206-18.

[24] Patterson F, Schnoll RA, Wileyto EP, Pinto A, Epstein LH, Shields PG, et al. Toward personalized therapy for smoking cessation: a randomized placebocontrolled trial of bupropion. Clin Pharmacol Ther 2008;84(3):320-5.

[25] Piper ME, Smith SS, Schlam TR, Fiore MC, Jorenby DE, Fraser D, et al. A randomized placebo-controlled clinical trial of 5 smoking cessation pharmacotherapies. Arch Gen Psychiatry 2009;66(11):1253-62.

[26] Jarvis MJ, Tunstall-Pedoe H, Feyerabend C, Vesey C, Saloojee Y. Comparison of tests used to distinguish smokers from nonsmokers. Am J Public Health 1987;77(11):1435-8, doi:http://dx.doi.org/10.2105/AJPH.77.11.1435.

[27] Heatherton TF, Kozlowski LT, Frecker RC, Fagerström KO. The Fagerström test for nicotine dependence: a revision of the Fagerstrom Tolerance Questionnaire. Br J Addict 1991;86(9):1119-27, doi:http://dx.doi.org/10.1111/ j.1360-0443.1991.tb01879.x.

[28] Sarbandi F, Niknami S, Hidarnia A, Hajizadeh E, Azaripour MH, Eslampanah NS. Psychometric properties of the Iranian version of the fagerstrom test for nicotine dependence and of heaviness of smoking index. J Res Health 2015;5 (1):96-103.

[29] Wall MA, Johnson J, Jacob P, Benowitz NL. Cotinine in the serum, saliva, and urine of nonsmokers, passive smokers, and active smokers. Am J Public Health 1988;78(6):699-701.

[30] Cooke F, Bullen C, Whittaker R, McRobbie H, Chen MH, Walker N. Diagnostic accuracy of NicAlert cotinine test strips in saliva for verifying smoking status. Nicotine Tob Res 2008;10(4):607-12, doi:http://dx.doi.org/10.1080/ 14622200801978680.

[31] Hurt RD, Sachs DP, Glover ED, Offord KP, Johnston JA, Dale LC, et al. A comparison of sustained-release bupropion and placebo for smoking cessation. N Engl J Med 1997;337(17):1195-202, doi:http://dx.doi.org/ 10.1056/NEJM199710233371703.

[32] Ahluwalia JS, Harris KJ, Catley D, Okuyemi KS, Mayo MS. Sustained-release bupropion for smoking cessation in African Americans: a randomized controlled trial. JAMA 2002;288(4):468-74, doi:http://dx.doi.org/10.1001/ jama.288.4.468.

[33] Scheuermann TS, Richter KP, Rigotti NA, Cummins SE, Harrington KF, Sherman $\mathrm{SE}$, et al. Accuracy of self-reported smoking abstinence in clinical trials of hospital-initiated smoking interventions. Addiction 2017;112(12):2227-36, doi:http://dx.doi.org/10.1111/add.13913.

[34] Diana M. The dopamine hypothesis of drug addiction and its potential therapeutic value. Front Psychiatry 2011;2(64):1-7, doi:http://dx.doi.org/ 10.3389/fpsyt.2011.00064.

[35] Nitsche MA, Lampe C, Antal A, Liebetanz D, Lang N, Tergau F, et al. Dopaminergic modulation of long-lasting direct current-induced cortical excitability changes in the human motor cortex. Eur J Neurosci 2006;23 (6):1651-7, doi:http://dx.doi.org/10.1111/j.1460-9568.2006.04676.x.

[36] Nitsche MA, Fricke K, Henschke U, Schlitterlau A, Liebetanz D, Lang N, et al. Pharmacological modulation of cortical excitability shifts induced by transcranial direct current stimulation in humans. J Physiol 2003;553(1):293301, doi:http://dx.doi.org/10.1113/jphysiol.2003.049916.

[37] Bindman LJ, Lippold OC, Redfearn JW. The action of brief polarizing currents on the cerebral cortex of the rat (1) during current flow and (2) in the production of long-lasting after-effects. J Physiol 1964;172(3):369-82, doi:http://dx.doi. org/10.1113/jphysiol.1964.sp007425.

[38] Bindman LJ, Lippold OC, Redfearn JW. Relation between the size and form of potentials evoked by sensory stimulation and the background electrical activity in the cerebral cortex of the rat. J Physiol 1964;171(1):1-25, doi:http:// dx.doi.org/10.1113/jphysiol.1964.sp007358.

[39] Nitsche MA, Nitsche MS, Klein CC, Tergau F, Rothwell JC, Paulus W. Level of action of cathodal DC polarisation induced inhibition of the human motor cortex. Clin Neurophysiol 2003;114(4):600-4, doi:http://dx.doi.org/10.1016/ S1388-2457(02)00412-1.

[40] Jansen JM, Daams JG, Koeter MW, Veltman DJ, van den Brink W, Goudriaan AE. Effects of non-invasive neurostimulation on craving: a meta-analysis. Neurosci Biobehav Rev 2013;37(10):2472-80, doi:http://dx.doi.org/10.1016/j. neubiorev.2013.07.009.

[41] Pedron S, Monnin J, Haffen E, Sechter D, Van Waes V. Repeated transcranial direct current stimulation prevents abnormal behaviors associated with abstinence from chronic nicotine consumption. Neuropsychopharmacology 2014;39(4):981-8, doi:http://dx.doi.org/10.1038/npp.2013.298.

[42] Liebetanz D, Nitsche MA, Tergau F, Paulus W. Pharmacological approach to the mechanisms of transcranial DC-stimulation-induced after-effects of human motor cortex excitability. Brain 2002;125(10):2238-47, doi:http://dx.doi.org/ 10.1093/brain/awf238.

[43] Nitsche MA, Paulus W. Sustained excitability elevations induced by transcranial DC motor cortex stimulation in humans. Neurology 2001;57 (10):1899-901, doi:http://dx.doi.org/10.1212/WNL.57.10.1899.

[44] Shiffman S, Gnys M, Richards TJ, Paty JA, Hickcox M, Kassel JD. Temptations to smoke after quitting: a comparison of lapsers and maintainers. Health Psychol 1996;15(6):455.

[45] Shiffman S, Waters AJ. Negative affect and smoking lapses: a prospective analysis. J Consult Clin Psychol 2004;72(2):192-201.

[46] Lerman C, Roth D, Kaufmann V, Audrain J, Hawk L, Liu A, et al. Mediating mechanisms for the impact of bupropion in smoking cessation treatment. 
Drug Alcohol Depend 2002;67(2):219-23, doi:http://dx.doi.org/10.1016/ S0376-8716(02)00067-4

[47] Wing VC, Barr MS, Wass CE, Lipsman N, Lozano AM, Daskalakis ZJ, et al. Brain stimulation methods to treat tobacco addiction. Brain Stimul 2013;6(3):22130, doi:http://dx.doi.org/10.1016/j.brs.2012.06.008.

[48] Peña-Gómez C, Vidal-Piñeiro D, Clemente IC, Pascual-Leone Á, Bartrés-Faz D. Down-regulation of negative emotional processing by transcranial direct current stimulation: effects of personality characteristics. PLoS One 2011;6(7): e22812, doi:http://dx.doi.org/10.1371/journal.pone.0022812.

[49] Nitsche MA, Koschack J, Pohlers H, Hullemann S, Paulus W, Happe S. Effects of frontal transcranial direct current stimulation on emotional state and processing in healthy humans. Front Psychiatry 2012;3:, doi:http://dx.doi.org/ 10.3389/fpsyt.2012.00058

[50] Fecteau S, Knoch D, Fregni F, Sultani N, Boggio P, Pascual-Leone A. Diminishing risk-taking behavior by modulating activity in the prefrontal cortex: a direct current stimulation study. J Neurosci 2007;27(46):12500-5, doi:http://dx.doi. org/10.1523/JNEUROSCI.3283-07.2007.

[51] Fecteau S, Agosta S, Hone-Blanchet A, Fregni F, Boggio P, Ciraulo D, et al. Modulation of smoking and decision-making behaviors with transcranial direct current stimulation in tobacco smokers: a preliminary study. Drug Alcohol Depend 2014;140:78-84, doi:http://dx.doi.org/10.1016/j. drugalcdep.2014.03.036.

[52] Rodriguez E, George N, Lachaux JP, Martinerie J, Renault B, Varela FJ. Perception's shadow: long-distance synchronization of human brain activity. Nature 1999;397(6718):430.

[53] Gersner R, Kravetz E, Feil J, Pell G, Zangen A. Long-term effects of repetitive transcranial magnetic stimulation on markers for neuroplasticity: differential outcomes in anesthetized and awake animals. J Neurosci 2011;31(20):7521-6, doi:http://dx.doi.org/10.1523/JNEUROSCI.6751-10.2011. 